\title{
A CONTROL STRATEGY OF A MOBILE ROBOT IN A LIMITED SPACE USING A LASER RANGEFINDER
}

\author{
S. JATSUN, O. EMELYANOVA, P. BEZMEN, A. S. MARTINEZ LEON, L. M. MOSQUERA \\ MOROCHO and D. AFONIN \\ Department of mechanics, mechatronics and robotics, Southwest State University \\ Kursk, 305040, Russian Federation \\ E-mail:newteormeh@inbox.ru
}

\begin{abstract}
Currently, one of the most promising areas of research in the field of robotics is related to the task of creating intelligent autonomous mobile robots able to move in a limited space, bypass stationary or moving obstacles, take by themselves optimal decisions based on the data obtained from measuring devices installed on the board of the robot, as well as build a map of the surrounding area, etc. This article deals with the development of a method for solving the problem of simultaneous localization and mapping (SLAM) by the use of a self-developed mobile platform in conditions of limited space, an algorithm of control for the mobile platform has been proposed, a visualization software to visualize the construction of the map by the robot in real time has been reached, an experiment to evaluate the accuracy of the system has been carried out.
\end{abstract}

Keywords: mobile robot, intelligent control systems, laser rangefinder, mapping, SLAM method

\section{Introduction}

The stage of development of control systems most determines the advances in robotics. In particular, there are several well-known methods in the field of trajectory control of mobile robots in a limited space such as the presented by different scientists like Yu. G. Martynenko [1], A.V. Lyamin [2], A.V. Timofeev [3], G. Bastin [4], etc. in their works. The essential feature of these methods is related to their requirement to access to the whole information about the parameters of the environment.

However, the information about the environment is often minimal or completely missing. It is a common situation when the environment is under the influence of stochastic or man- made factors, or contains mobile objects that move arbitrary. In this case, it becomes necessary to solve the task of controlling the movement of a mobile robot in a non-deterministic environment. In this case, the algorithm of control in development for a mobile robot must satisfy properties such as adaptiveness, self-learning, and capability of handling uncertainties [2,5, 16,19].

Nowadays, despite the several publications in the field of intelligent control methods for mobile robots, in most cases, the implementation of algorithms of control for the movement of mobile robots in non-deterministic conditions is associated with a large number of difficulties such as the complexity of synthesizing the whole information about the environment. Thus, it results that it is necessary to use a low-level control method, i.e. to control a mobile robot using a remote control from a remote station by an operator. This approach has significant disadvantages such as: limited scope of mobile robots, which is related with the need to maintain the communication between the robot and the remote station; the complexity of the commands needed for controlling the robot, which leads to operator fatigue; and also the complexity implementing an accurate control method based on telemetry data $[2,7,8,17,20]$. 
In order to exclude these disadvantages, it is necessary to develop intelligent control systems capable to provide an autonomous work of a mobile robot. In this case, the participation of the operator is necessary at the setting level of the goal.

The main advantage of intelligent mobile robots is related to the ability to navigate in an autonomous way through an unknown space. The robot should be able to estimate its position, recognize and store the necessary information about the environment. Thus, it becomes possible to build a map of the environment and solve different required tasks by the robot in a specified area of the environment. This process is solved using a method for Simultaneous Localization and Mapping (SLAM) [9,10,13,15-20].

During these years, several approaches related to the development and implementation of different SLAM methods have been reached by scientist around the world. According to D.A. Baramiya, M.S. Dyakov, M.M. Lavrentiev [5] it is proposed to classify the existing SLAM methods as follows:

- $\quad$ By type of measuring and optical devices: RGB-D cameras [11,15], two-dimensional laser rangefinders [10], etc.

- By calculation methods (using an algorithm for constructing hypotheses about the current position of the robot): Kalman filter [7,12,14], particle filter [12], etc.

The existing SLAM approaches are based on modern and complex computer algorithms. Its implementation depends on the hardware and software capabilities of the platforms on which they are realized.

In this paper a method for simultaneous localization and mapping called SLAM-LS method has been proposed. In order to implement this method, a laser rangefinder has been installed onboard the mobile platform in use. With the aim to obtain a suitable information about the environment, the data received from the lidar is processed and filtered. At this stage, the operator from a ground station is able to visualize the construction of the map by the robot in real time.

This paper is structured as follows: Section 2 presents a brief description of structure of the proposed intelligent mobile platform for implementing the introduced SLAM-LS algorithm; Section 3 describes the introduced SLAM-LS method based on the Kalman filter (KF); Section 4 presents a description of the data filtering algorithm, Section 5 contains the main experimental results obtained in this work; Section 6 concludes this purpose, such as some notes about future work.

\section{Statement of the problem}

In some cases, a mobile robot controlled by an operator at the goal setting level must move in a non-deterministic environment characterized by a previously unknown location of the existing dynamical obstacles and targets. In these conditions is necessary to realize a dynamical analysis of the environment, where the robot is going to operate. This analysis is based on the information obtained from the measuring devices, and also from the selected goal by the operator, in this way becomes possible to reach a good performance in terms of navigation and control of the movement of the robot.

The task of self-realization (self-determination of the position) of a mobile robot is related to the task of modeling the environment, where the robot plans to operate, i.e. these solutions are mutually dependent. This can be solved using the methods of path planning, and also with the use of full and sensory localization. Often, the both methods are used in conjunction in order to perform a better result [6-8].

The aim of this paper is to introduce a modified SLAM-LS algorithm (Fig.1), which includes the following subtasks:

- Estimation of the current position of the robot based on the obtained data from the measuring devices in use.

- Determination of the obstacles and elements of the environment (information is saved as time points). 
- Association of new and previous data (comparation of new data with previous information or adding new points to the map).

- Updating and storing the map of the environment.

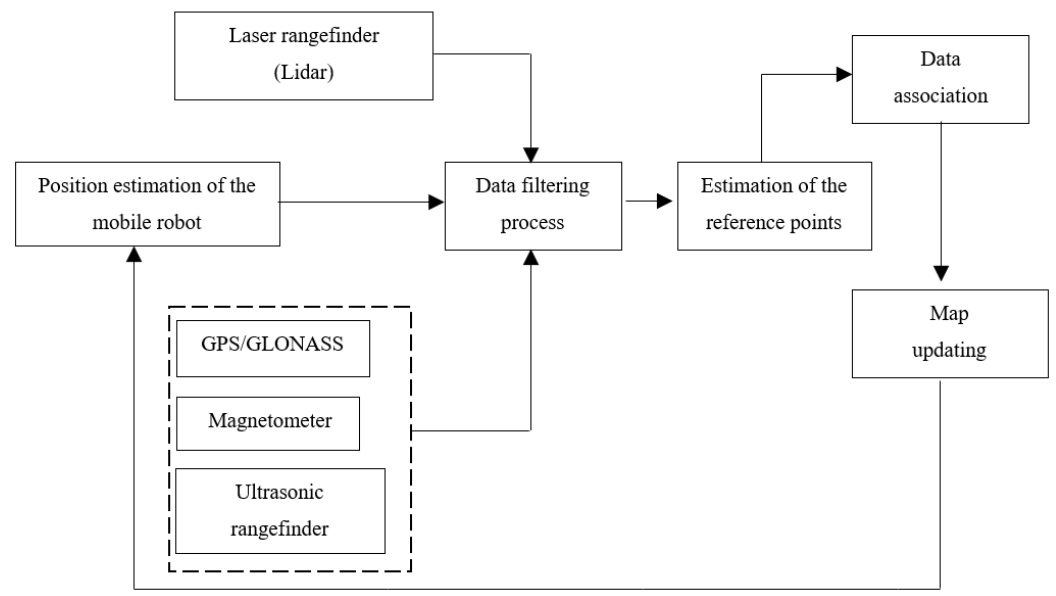

Fig.1. SLAM-LS algorithm scheme

The introduced navigation method for the movement of the mobile robot and its capability to solve the proposed SLAM task in terms of measuring devices involves the implementation of a global positioning (GLONASS and GPS) systems and a laser rangefinder (LIDAR), a magnetometer, and an ultrasonic rangefinder.

In order to achieve this approach, it is necessary to distinguish two important terms related to the navigation task of a mobile robot:

- Geometrical localization (estimates the position and orientation of the robot).

- Topological localization (a reference of the robot with respect to the environment).

With the aim to implement a control strategy of the mobile robot during its spatial movement it is necessary to introduce at least two types of coordinate systems: a local mobile coordinate system associated with the center of mass of the robot, and a global system associated with the origin of coordinates at some point in space (Fig. 2).

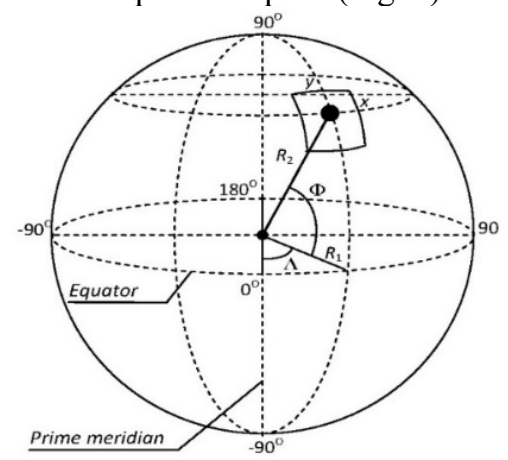

Fig.2. Spherical coordinates of the Earth

To provide the robot movement control process we need to convert spherical coordinates of the Earth (longitude and latitude) into rectangular coordinates $-x$ and $y$ :

$$
\begin{aligned}
& x=\Lambda \cdot R_{1}, \\
& y=\Phi \cdot R_{2},
\end{aligned}
$$

where: $\Lambda$ - longitude $(-\pi \leq \Lambda \leq \pi \mathrm{rad}), \Phi$ - latitude $(-\pi / 2 \leq \Phi \leq \pi / 2 \mathrm{rad})$, $R_{1}=6378.16 \mathrm{~km}$ (Equatorial radius), $R_{2}=6356.77 \mathrm{~km}$ (Polar radius). 
The inaccuracy of bearing $\varphi_{i}$ determination can be denoted by $\Delta \varphi$. Thus, during robot movement to a destination point the robot body executes process of rotation so that the bearing value could be $\varphi_{i} \pm \Delta \varphi$, where $\varphi_{i}$ is calculated on the basis of current coordinates $\left(x_{C}\right.$ and $\left.y_{C}\right)$ of the robot body and coordinates $\left(x_{i}\right.$ and $\left.y_{i}\right)$ of a destination point [5]:

$$
\varphi_{i}=\left\{\begin{array}{l}
{\left[\frac{\pi}{2}-\left[\arccos \frac{x_{i}-x_{C}}{\left[\sqrt{\left(x_{i}-x_{C}\right)^{2}+\left(y_{i}-y_{C}\right)^{2}}\right]}\right]\right] \text {, if }\left(y_{i}-y_{C}\right) \geq 0,} \\
\left.\pi \cdot \operatorname{sign}\left(x_{i}-x_{C}\right)+\left[\frac{\pi}{2}-\left[\arccos \left[\frac{x_{i}-x_{C}}{\left[\sqrt{\left(x_{i}-x_{C}\right)^{2}+\left(y_{i}-y_{C}\right)^{2}}\right] \cdot \operatorname{sign}\left(x_{i}-x_{C}\right)}\right]\right]\right] \cdot\left(-\operatorname{sign}\left(x_{i}-x_{C}\right)\right)\right], \text { if }\left(y_{i}-y_{C}\right)<0,
\end{array}\right.
$$

where sign is the signum function:

$$
\operatorname{sign}(x)=\left\{\begin{array}{l}
1, \quad \text { if } \quad x \geq 0, \\
-1, \quad \text { if } \quad x<0 .
\end{array}\right.
$$

Thereby, $\varphi_{i} \in(-\pi, \pi]$. Sign of the bearing $\varphi_{i}$ means a destination point location: at $\varphi_{i}<0$ destination point is located in the west, at $\varphi_{i}>0-$ it is located in the east.The course deviation is a difference between the specified bearing $\varphi_{i}$ and the current bearing $\varphi_{C}$ :

in this connection:

$$
\Theta=\varphi_{i}-\varphi_{C},
$$

$$
\begin{aligned}
& \text { if } \Theta>\pi \text {, then } \Theta=-(2 \pi-\Theta) \text {, } \\
& \text { if } \Theta \leq-\pi \text {, then } \Theta=2 \pi+\Theta \text {, } \\
& \qquad \Theta \in(-\pi, \pi] .
\end{aligned}
$$

After course deviation calculation there are possible the following variants of robot movement:

- if $|\Theta|>\Delta \varphi$ and $\Theta>0$, then the robot body turns to right (clockwise rotation);

- $\quad$ if $|\Theta|>\Delta \varphi$ and $\Theta<0$, then the robot body turns to left (contraclockwise rotation);

- if $|\Theta| \leq \Delta \varphi$, then the robot body does not turn, i.e. there is possible straightforward movement of the robot.

The criterion of robot movement completion can be denoted by following equations:

where:

$$
\left|x_{i}-x_{C}\right|=\Delta x,\left|y_{i}-y_{C}\right|=\Delta y,\left|\varphi_{i}-\varphi_{C}\right|=\Delta \varphi,
$$

- $\quad x_{i}, y_{i}$ and $\varphi_{i}$ are destination point coordinates and specified bearing;

- $\quad x_{C}, y_{C}$ and $\varphi_{C}$ are current robot body coordinates and bearing;

- $\Delta x, \Delta y$, and $\Delta \varphi$ are determination inaccuracies of coordinates $x, y$ (induced by GPS receiver bias and obstacles for GPS signals), and bearing $\varphi$ (induced by magnetometer and intense magnetic fields), respectively.

Generally, the $\Delta x$ and $\Delta y$ values are induced by lay of land and man-made structures which are obstacles for GPS signals. In many cases the $\Delta x$ and $\Delta y$ inaccuracies are independent and different values (Fig. 3). 


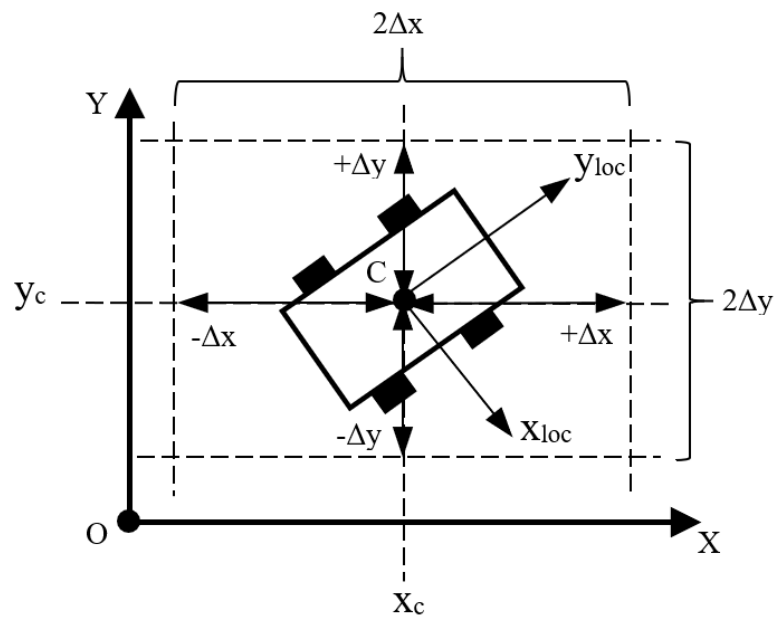

Fig. 3. $\Delta x$ and $\Delta y$ inaccuracies: $\mathrm{OXY}-$ global coordinate system, $\mathrm{x}_{\mathrm{C}}, \mathrm{y}_{\mathrm{C}}$ - robot coordinates in the global coordinate system, $\mathrm{Cx}_{\text {loc }} \mathrm{y}_{\text {loc }}$ - local coordinate system, $\mathrm{C}$ - robot mass center

\section{Description of the structure of the mobile robot}

The mobile robot "X4WD" (Fig. 4) was developed by the laboratory of mechanics, mechatronics and robotics at Southwest State University for the development and research of control methods for autonomous mobile platforms. The mobile platform is based on a fourwheel chassis frame. The "X4WD" is equipped with an intelligent control system, a power supply and a set of measuring devices. The robot's overall dimensions are 780 × $305 \times 340 \mathrm{~mm}$, its weight is $5 \mathrm{~kg}$, and the maximum linear speed when moving on a flat surface is $10 \mathrm{~km} / \mathrm{h}$.

The robot control system is based on the Arduino Due and Teensy 3.6 controllers. The mobile robot "X4WD" is a mobile remote-controlled system. Data transfer between the robot and the remote control is performed by the ZigBee transceiver. The map of the environment is saved in a microSD card inserted into the Teensy 3.6 card slot (Fig. 5).

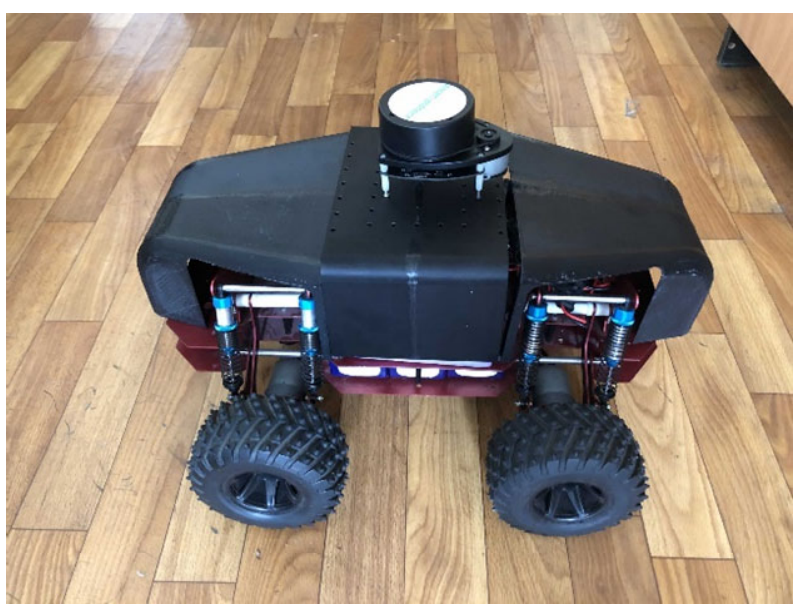

Fig.4. Prototype of the mobile robot "X4WD" 


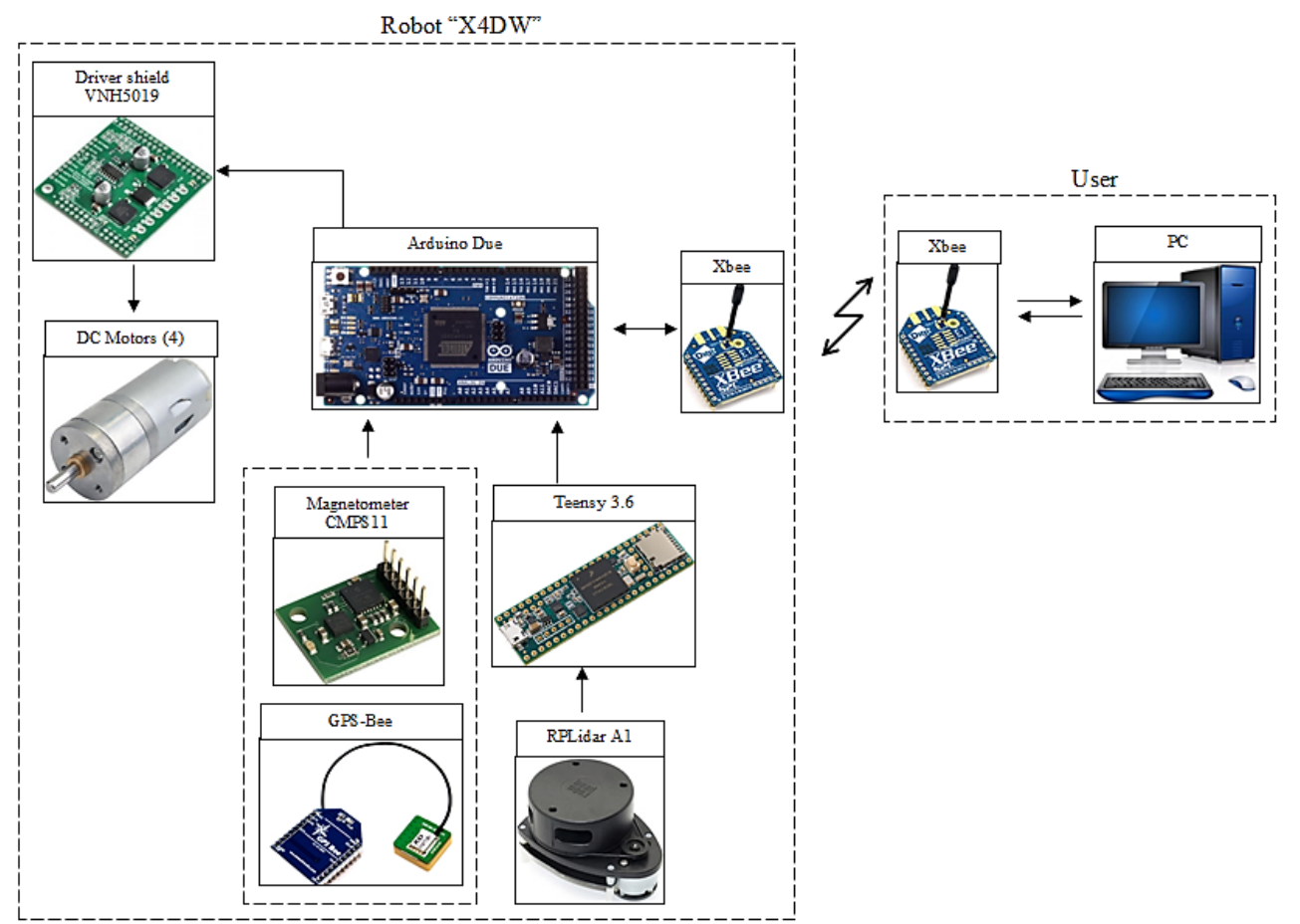

Fig.5. Scheme of the control system of the robot "X4WD"

For building the map of the environment with the use of the introduced SLAM-LS method, it is necessary to realize the following steps (Fig. 6):

- Getting data about the current latitude and longitude (geographical coordinates) of the mobile robot using the GPS/GLONASS signal receiver.

- Getting data about the current position of the robot and coordinates from the lidar (relative to the direction to the north magnetic pole using a magnetometer).

- Building a map using lidar and storing the resulting array of data in memory, i.e. accumulating information about the current obstacles surrounding the mobile robot: information about the angle of rotation of the lidar and corresponding data about the distance to the obstacle or the distance to the obstacles (in the case of moving obstacles).

- Data filtering using a the Kalman filter (KF);

- Correction of map data (turning the map by an angle equal to the rotation of the reference point of the lidar coordinates relative to the direction to the north magnetic pole).

- Assigning the current values of geographical coordinates to the coordinates of the robot and lidar.

- Merging geographical coordinates with the map data array, assigning each map point its own geographical coordinates in accordance with its distance from lidar.

- $\quad$ Saving the map to a memory card. 


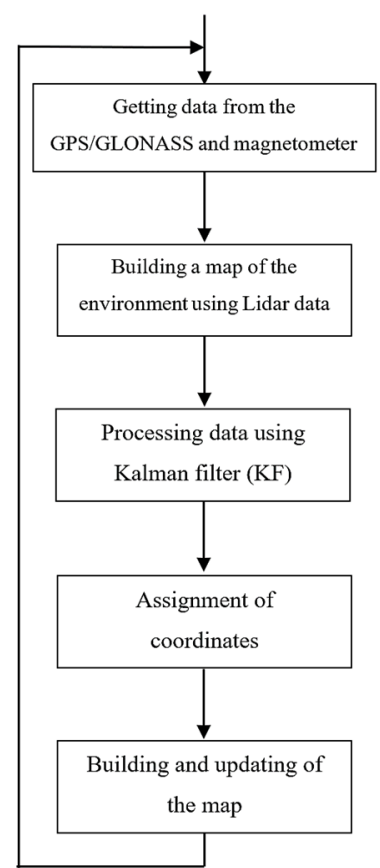

Fig.6. Mapping process using SLAM-LS method

\section{Processing data using the Kalman filter}

The Kalman Filter (KF) is one of the most widely used methods for tracking and estimation due to its simplicity, optimality, tractability and robustness. Different implementations of the Kalman filter are presented in works $[6,7,13]$. This method is widely used in navigation systems of aircrafts, ships, submarines, spacecrafts, missiles, ground vehicles, etc. For a long time, this recurrent filter had a limited application due to the high requirements imposed on the onboard equipment of the robot in terms of computing capability and a large amount of memory required from the microcontrollers and other devices.

The algorithm of implementation of Kalman filter can be divided into 4 stages: 1 initialization, 2 - prediction, 3 - observation, 4 - evaluation [14].

Stages $2-4$ of the algorithm run cyclically and make up each cycle of the filter; stages 3 and 4 can be combined into the correction stage (Fig. 7). At the initialization stage, elements of the arrays used in the algorithm (vectors and matrices) are assigned zero and initial values are set for elements of the noise covariance matrix. In the second stage, the state of a certain system is predicted at the next moment of time. The third stage is related to obtain new information (for example, data from a sensor). In the fourth stage, the obtained information corrects the predicted state values.

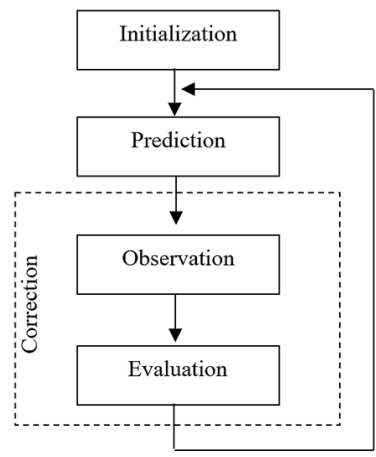

Fig. 7. Algorithm of implementation of Kalman filter (KF) 
In this work, we use this method in order to filter and add missing information about the state of the robot for building a map of the environment. In Fig. 8 is shown the processing method used for the information obtained from the Lidar.

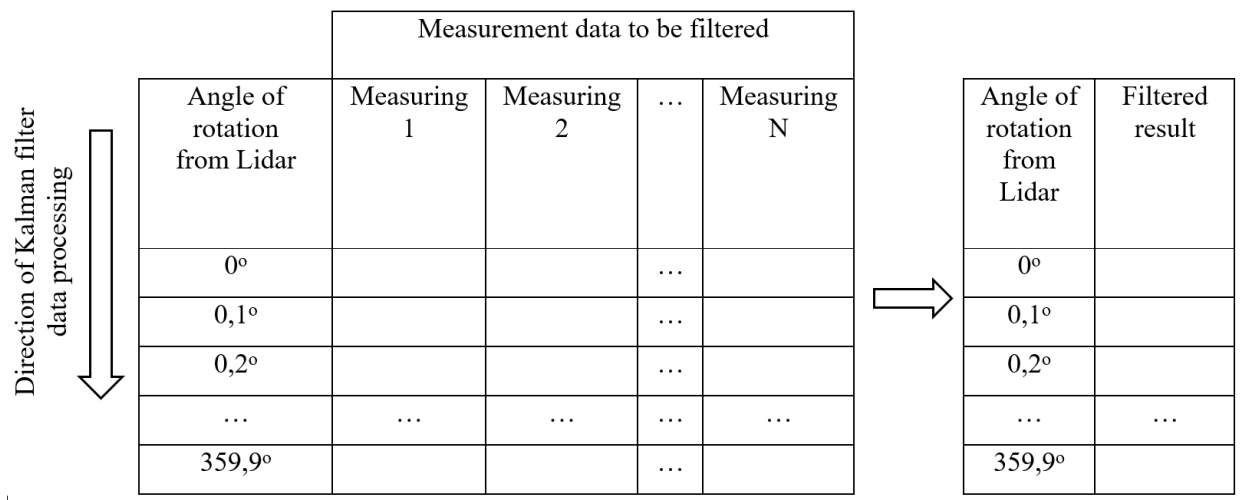

Fig.8. Processing method of the information from the Lidar

\section{Experimental results}

With the aim to test the accuracy and effectiveness of the introduced SLAM-LS algorithm an experiment has been carried out in the premises of the Department of mechanics, mechatronics and robotics at Southwest state University.

To visualize the results, a software called "Terminal" has been developed. This software is able to realize real- time tracking of the movement of the mobile platform in the environment, as well as to build the map of a certain area in study, based on the information obtained from the measuring devices and the implemented algorithms. The main results of the experiment are presented in Fig. 9-10.

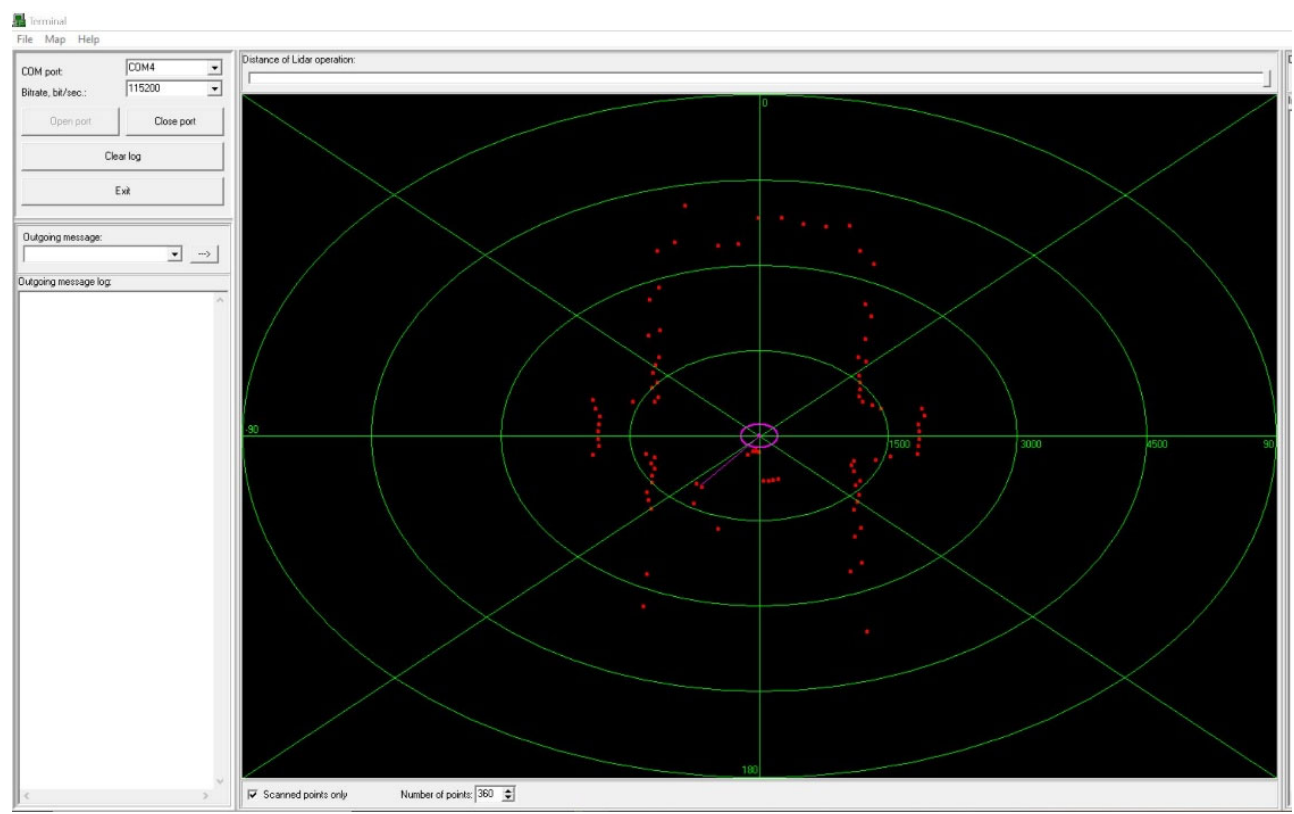

Fig.9. Visualization of the robot's perception of the environment 


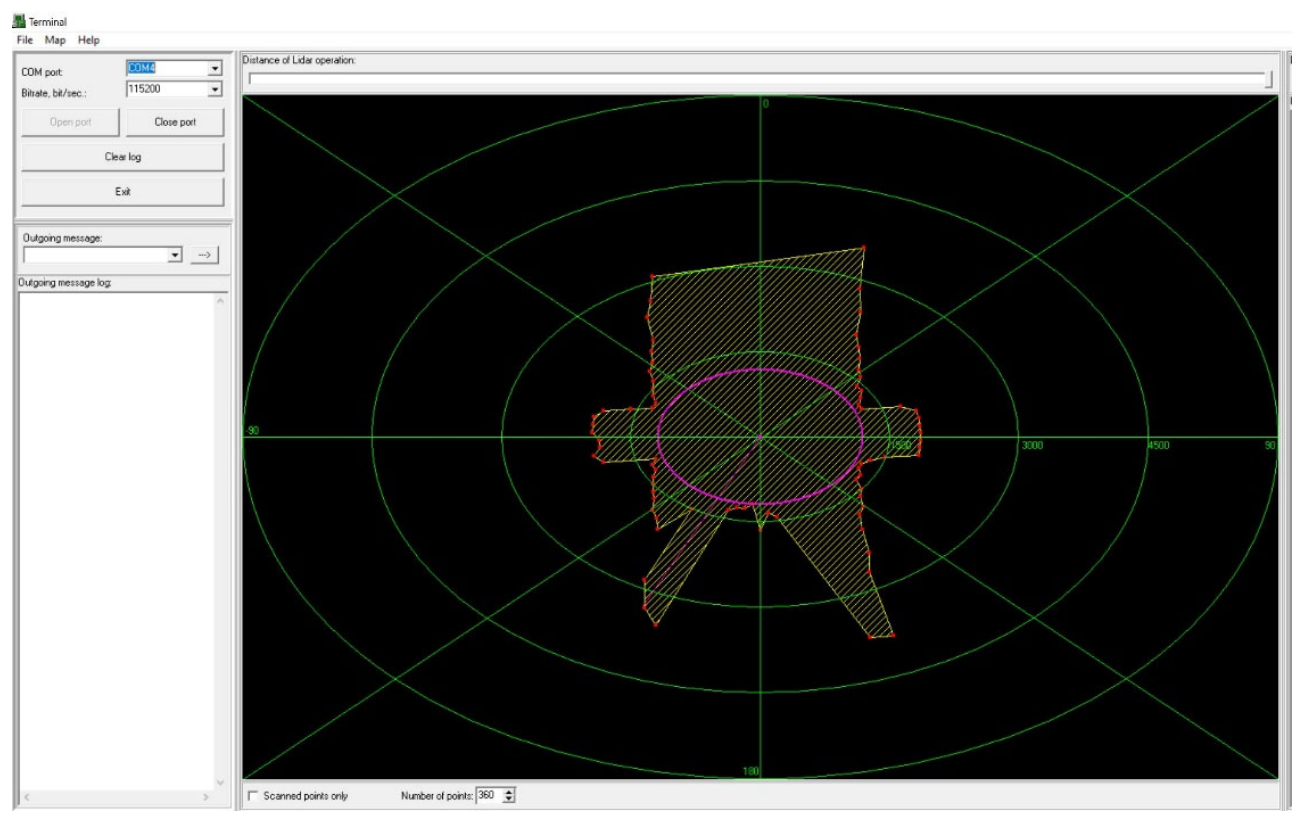

Fig.10. Visualization of the map of the environment

\section{Conclusions and future work}

In this paper the main existing methods for controlling mobile robots in a limited space have been analyzed. A method for simultaneous localization and mapping called SLAM-LS method has been introduced. A strategy for autonomous control of the mobile robot "X4WD" has been carried out. The proposed method involves the use of a laser rangefinder installed in the robot. The received data from the lidar is processed and filtered, creating an array of values corresponding to the distances (in meters) from the lidar to the surface of obstacles located in the range of view of the laser rangefinder. The values of the array are ordered according with the angle of the scanning angle, which changes from $0^{\circ}$ to $+359.9^{\circ}$ with an increment of $0.1^{\circ}$, its size is equal to 3600 measurements. The maximum measurement range (scanner visibility limit) is $5 \mathrm{~m}$. A software to visualize the construction of the map by the robot in real time has been developed, an experiment to evaluate the accuracy and effectiveness of the strategy of control for the mobile robot "X4WD", as well as the introduced SLAM-LS method has been carried out. Further, there is going to be integrated a stereo camera type Kinect in order to extend the presented SLAM-LS algorithm for 3D reconstruction of an specify environment, tracking, recognition and surveillance of static or dynamical targets.

\section{References}

1. Yu. G. Martynenko. Upravlenie dvizheniem mobil'nyh kolesnyh robotov. Journal "Fundamentalnaya i Prikladnaya Matematika", 2005, 8, pp. 29-80 (in Russian).

2. G.I. Boltunov, A.V. Lyamin, A.I. Petrik. Algoritm upravleniya dvizheniem mobil'nogo kolesnogo robota $\mathrm{v}$ zadache slezheniya za ekzosistemoj. Journal "Proceedings of Higher Educational Institutions”, 2013, 4(56), pp. 71-75 (in Russian).

3. A.V. Timofeev. Multiagent robotic systems and neural network technologies. Journal "Izvestiya SFedU. Engineering sciences", 2010, 3(104), pp 20-23 (in Russian).

4. B. D'Andréa-Novel, G. Campion, G. Bastin. Control of nonholonomic wheeled mobile robots by state feedback linearization. International journal of robotics research, 1995, 6(14), pp. 543-559. 
5. D.A. Baramiya, M.S. D'yakov, M.M. Lavrent'ev. Development of simultaneous localization and mapping system based on lidar and cameras data. Journal "NSU. Series: Information Technologies", 2015, 1(13), pp 5-15 (in Russian).

6. Y. Bar-Shalom, X. R. Li, T. Kirubarajan. Estimation with applications to tracking and navigation: theory algorithms and software. John Wiley \& Sons (2004).

7. P. A. Bezmen. Integration of Mobile Robot Control System Data Using the Extended Kalman Filter. In Proceedings of the Southwest State University, 2019, 2(23), pp. 5364.

8. S.F. Jatsun, et al. Control system of the independent undersea robot. In 2nd International Research and Practice Conference, 2012, 1, pp. 165-170.

9. M. Osman, A. Hussein, A. Al-Kaff. Intelligent Vehicles Localization Approaches between Estimation and Information: A Review. In 2019 IEEE International Conference of Vehicular Electronics and Safety (ICVES), 2019, pp. 1-8.

10. A. Yu. Filatov, et al. Comparison of modern SLAM laser algorithms. Journal MIR (Modernization. Innovation. Research), 2001, 412, pp. 66-73 (in Russian).

11. C. Kerl, J. Sturm, D. Cremers. Dense visual SLAM for RGB-D cameras. In 2013 IEEE/RSJ International Conference on Intelligent Robots and Systems, 2013, pp. 2100-2106.

12. F. Zhang et al. Algorithms analysis of mobile robot SLAM based on Kalman and particle filter. In 9th IEEE International Conference on Modelling, Identification and Control (ICMIC), 2017, pp. 1050-1055.

13. М.И. Sobchenko, et al. Algoritmy SLAM: obzor sushchestvuyushchih reshenij. Journal "Electronic Information Systems", 2014, 1, pp. 69-78.

14. X. Jiang, et al. A novel SLAM algorithm with Adaptive Kalman filter. In IEEE International Conference on Advanced Robotics and Mechatronics (ICARM), 2016, pp. 107-111.

15. R. A. Newcombe, et al. KinectFusion: Real-time dense surface mapping and tracking. In 10th IEEE International Symposium on Mixed and Augmented Reality, 2011, pp. $127-136$

16. R. Ren, H. Fu, M. Wu. Large-scale outdoor slam based on 2 d lidar. Journal "Electronics", 2019, 6(8), pp. 613.

17. G. Jiang, et al. A simultaneous localization and mapping (SLAM) framework for 2.5 D map building based on low-cost LiDAR and vision fusion. Journal "Applied Sciences", 2019, 9(10), pp. 2105.

18. Z. Cui, et al. Optimal Parameter Analysis of Two 2D Lidar SLAM. Journal "World Scientific Research Journal", 2020, 1(6), pp. 56-62.

19. C. Debeunne, D. Vivet. A Review of Visual-LiDAR Fusion based Simultaneous Localization and Mapping. Journal "Sensors", 2020, 7(20), pp. 2068.

20. C. Xin, et al. Research on Indoor Navigation System of UAV Based on LIDAR. In IEEE 12th International Conference on Measuring Technology and Mechatronics Automation (ICMTMA), 2020, pp. 763-766. 